\title{
p38 MAPKs - roles in skeletal muscle physiology, disease mechanisms, and as potential therapeutic targets
}

\author{
Christopher M. Brennan, ${ }^{1}$ Charles P. Emerson Jr., ${ }^{2}$ Jane Owens, ${ }^{1}$ and Nicolas Christoforou ${ }^{1}$ \\ ${ }^{1}$ Rare Disease Research Unit, Pfizer Inc., Cambridge, Massachusetts, USA. ${ }^{2}$ Wellstone Muscular Dystrophy Program, \\ Department of Neurology, University of Massachusetts Medical School, Worcester, Massachusetts, USA.
}

p38 MAPKs play a central role in orchestrating the cellular response to stress and inflammation and in the regulation of myogenesis. Potent inhibitors of p38 MAPKs have been pursued as potential therapies for several disease indications due to their antiinflammatory properties, although none have been approved to date. Here, we provide a brief overview of p38 MAPKs, including their role in regulating myogenesis and their association with disease progression. Finally, we discuss targeting p38 MAPKs as a therapeutic approach for treating facioscapulohumeral muscular dystrophy and other muscular dystrophies by addressing multiple pathological mechanisms in skeletal muscle.

Conflict of interest: $\mathrm{CMB}, \mathrm{JO}$, and NC are employees of Pfizer Inc. J0 and NC also have ownership in Pfizer Inc.

Copyright: (c) 2021, Brennan et al. This is an open access article published under the terms of the Creative Commons Attribution 4.0 International License.

Reference information: /CI Insight. 2021;6(12):e149915.

https://doi.org/10.1172/jici. insight.149915.

\section{p38 family of MAPKs}

Mitogen-activated protein kinases (MAPKs) are serine/threonine kinases that are members of conserved signaling pathways that alter cellular physiology in response to extracellular signals (for review, see ref. 1). ERK, JNK, and p38 are major MAPKs in mammals, and like all MAPKs, are activated following a series of phosphorylation events. In response to external cues, MAPK kinase kinases (MAPKKKs, or MAP3Ks) phosphorylate MAPKKs (MAP2Ks) that then phosphorylate and activate MAPKs (Figure 1). MAPK signaling activity is regulated by cellular phosphatases that remove the activating phosphate groups. MAPK substrates include regulatory hubs, such as transcription factors and other signaling kinases, that control a variety of cellular processes, including cell division, inflammation, and differentiation.

The p38 MAPK family is composed of 4 related kinases, p38 $\alpha$ (also known as MAPK14), p38 $\beta$ (MAPK11), p38 (MAPK12), and p38ס (MAPK13; refs. 2, 3). p38 $\alpha$ is ubiquitously expressed in human tissues, whereas expression of $\mathrm{p} 38 \beta$ and $\mathrm{p} 38 \gamma$ is largely restricted to brain and muscle, respectively (4). The 4 p38 family members are $60 \%$ conserved at the amino acid level and have largely overlapping substrates (3). Due to their similarity, many of the inhibitors and antibodies used in studies described in this Review are incapable of distinguishing between different p38 family members; however, distinctions are made here when possible. p38 MAPKs contain an N-terminal and C-terminal domain, with the ATP-binding site located at the junction of the 2 domains (5), and interact with substrates via docking sites in their C-terminal domain (6). These MAPKs are strongly activated by cytokines and cellular stress, which may arise due to osmotic, oxidative, UV light, and heat shock stress. These kinases can, however, be activated - albeit to a lesser extent and more transiently - by homeostatic functions, including myogenesis, as discussed in detail below. Three MAP2Ks (MKK3, MKK4, and MKK6) regulate p38 activity. MKK4 specifically regulates p38 $\alpha$ and p38 $\beta$; MKK6 is

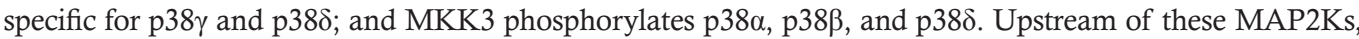
several MAP3Ks regulate $\mathrm{p} 38$ signaling, and some respond to specific stresses. For example, ASK1 activates p38 in response to oxidative stress (7), while MEKK1 activates the pathway in response to UV radiation (8); thus, p38 kinases are able to integrate multiple external cues to control cellular functions.

Much of what has been learned about the substrates and function of p38 comes from studies with inhibitors of these kinases. Most p38 inhibitors bind within the ATP-binding pocket of the active site, competitively blocking ATP binding, thus inhibiting kinase activity (9). Due to the high degree of amino acid similarity in their active sites, many p38 inhibitors target both p38 $\alpha$ and p38 $\beta$ (10). These inhibitors were first identified in phenotypic screens due to their ability to prevent cytokine production by LPS-stimulated human monocytes in vitro (11-13). Although several p38 $\alpha / \beta$ inhibitors have been tested in clinical trials for a variety of inflammatory diseases (e.g., acute coronary syndrome, chronic obstructive pulmonary disease [COPD], and rheumatoid arthritis [RA]), none have yet gained regulatory approval due to lack of efficacy (14), as further discussed below. 


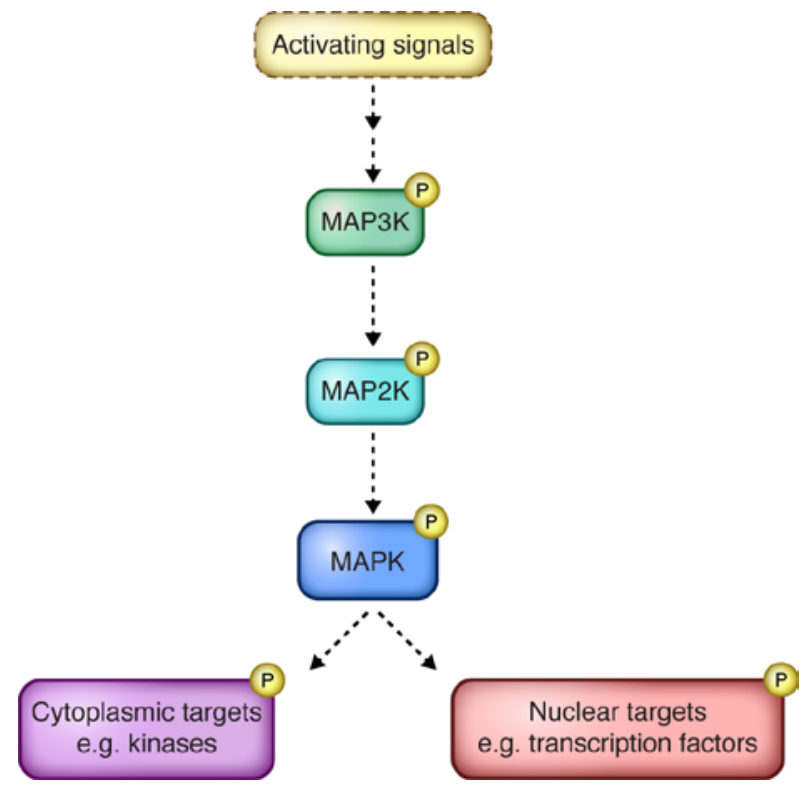

Figure 1. MAPK signaling cascade. Canonical mitogen MAPK signaling begins with an external activating signal, such as growth factors or stress, and proceeds through a series of activating phosphorylation events. MAPK kinase kinases (MAP3Ks) phosphorylate MAPK kinases (MAP2Ks), which phosphorylate MAPKs. Activated MAPKs facilitate the cellular response to the external signal by phosphorylating both cytoplasmic and nuclear proteins. These targets can include other central regulatory hubs, including other kinases and transcription factors. Illustrated by Rachel Davidowitz.

p38 $\alpha$, p38ß and p38 have well-established roles in skeletal myogenesis and muscle function, as recently reviewed by Segalés, et al. (15). Here, we explore the role of p38 in regulating skeletal muscle homeostasis, the disease process, and $\mathrm{p} 38 \alpha / \beta$ targeting as an approach to treating muscular dystrophies, such as facioscapulohumeral muscular dystrophy (FSHD), that is currently undergoing clinical testing.

\section{Role of p38 kinases in skeletal myogenesis}

The health of skeletal muscle depends on the maintenance of a robust population of quiescent muscle stem cells, called satellite cells, and their ability to become activated and differentiate to replace injured or diseased muscle. p38 kinases have multiple roles in this process, some of which are highlighted below. For in-depth review, see refs. 15 and 16.

p38 in myogenic differentiation. Myogenesis is the process by which activated satellite cells form proliferating myoblasts that differentiate and fuse to form multinucleated myofibers that constitute functional skeletal muscle. This process is controlled by basic helix-loop-helix (bHLH) transcription factors, namely Myf5, MyoD, myogenin, and MRF4, collectively referred to as myogenic regulatory factors (MRFs; ref. 17). Sequential expression and activation of MRFs promote progression of satellite cells through myogenesis. $\mathrm{MyoD}$ and Myf5 are expressed in uncommitted myoblasts; myogenin is expressed during early differentiation; and MRF4 is expressed during late differentiation $(16,18)$. MRFs dimerize with another class of bHLH transcription factors, Id (inhibitor of DNA binding) proteins, and with E proteins. Id proteins lack DNA-binding domains, and thereby inhibit transcription by MRFs (19). Downregulation of Id proteins at the onset of differentiation releases inhibition of MRFs, allowing for their activation (18). Heterodimers of MRFs and E proteins bind specific sequence elements (E boxes) within enhancers and promoters of genes involved in muscle differentiation and function (19). Additionally, proteins in the myogenic enhancer factor 2 (MEF2) family associate with MRFs and further enhance their transcriptional specificity and activity to express muscle protein-encoding genes (20).

p38 is expressed and has an active role at the onset of differentiation. Multiple studies have reported its involvement in regulating MRF transcription of muscle protein genes during skeletal muscle differentiation (2123) (Figure 2). First, p38 $\alpha$ directly phosphorylates the MEF2 coactivator of $\mathrm{MyoD}$ to promote the formation of a functional MyoD-MEF2 heterodimer $(24,25)$ (Figure 2). Similarly, p38 $\alpha / \beta$ phosphorylates E47 (also known as TCF3) to promote the formation of an active a MyoD-E47 heterodimer (26) (Figure 2). p38 $\alpha$ also promotes myotube formation independently of MRFs by upregulating expression of the tetraspanin protein CD53, which localizes to the cell membrane and facilitates efficient myoblast fusion and myofiber formation (27). p38 $\alpha$ also 


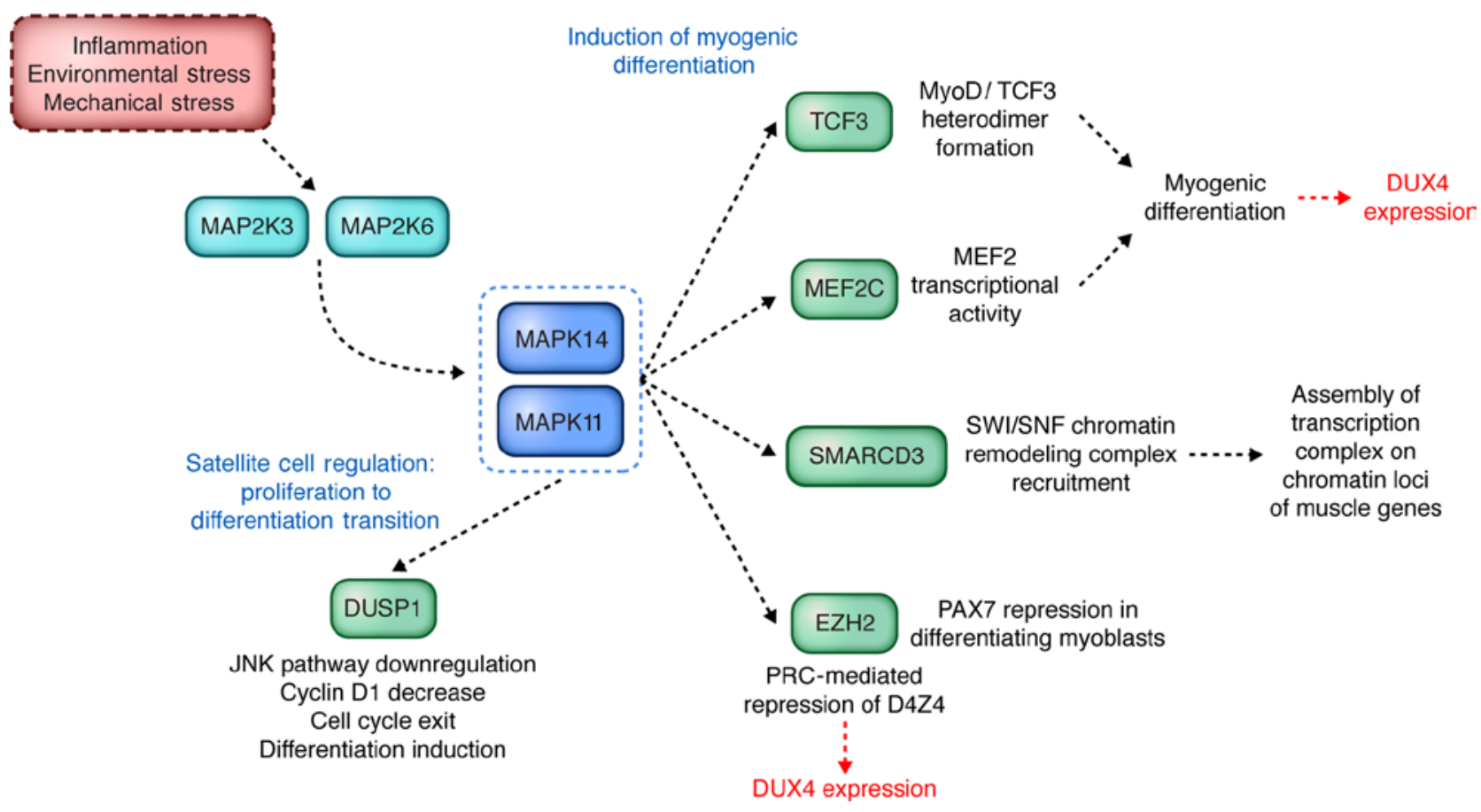

Figure 2. Roles of p38 MAPK in myogenic differentiation and satellite cell function for regulation of the FSHD disease gene DUX4. Members of the p38 family of MAP kinases are activated in response to stress but also to a lesser extent in muscle cells by the myogenic differentiation program. p38 $\alpha$ (MAPK14) and p38 $\beta$ (MAPK11) can be activated by 2 different MAP2Ks, MAP2K3 (MKK3) and MAP2K6 (MKK6). p38 $\alpha$ and p38 $\beta$ have both been shown to have roles in regulation of satellite cell fate and myogenic differentiation; however, the use of inhibitors that are potent against both p38 $\alpha$ and p38 $\beta$ in many studies makes discerning their roles independent of one another difficult. Examples of the role of $\mathrm{p} 38 \alpha / \beta$ in regulation of myogenesis are illustrated here and discussed in more detail in the main text. Potential points of DUX4 regulation by p38 are indicated in red. Illustrated by Rachel Davidowitz.

localizes to muscle gene regulatory elements bound by MRFs during differentiation, and $\mathrm{p} 38 \alpha / \beta$ inhibitors prevent recruitment of the chromatin remodeling complex SWI/SNF to these loci (28). This recruitment is likely mediated through direct phosphorylation of BAF60C (also known as SMARCD3), a subunit of SWI/SNF reported to be involved in its interaction with transcription factors $(28,29)$ (Figure 2). Thus, p38 $\alpha / \beta$ can promote transcription of myogenic genes by recruiting SWI/SNF to their regulatory elements, allowing the formation of euchromatin. Recent studies have shown that $\mathrm{p} 38 \alpha$ binds to both active and inactive promoters at the onset of differentiation, further highlighting a role in transcription regulation, though whether or not p38 activity directly contributes to transcriptional repression has not been determined (30). p38 $\alpha / \beta$ can also regulate the myogenic gene program posttranscriptionally via the RNA-binding protein KSRP, which binds RNA and promotes its degradation. During differentiation, KSRP is phosphorylated in a p38 $\alpha / \beta$-dependent manner, preventing it from binding and degrading myogenic transcripts. Thus, $\mathrm{p} 38 \alpha / \beta$ effectively stabilizes myogenic transcripts (31). Additionally, there is evidence for crosstalk between signaling pathways activated by p38 $\alpha / \beta$ and the MAPK family member JNK that promotes cell proliferation (Figure 1). Murine myoblasts derived from neonatal skeletal muscle genetically engineered to lack p38 $\alpha$ fail to exit the cell cycle, even in differentiation-promoting conditions, and this coincides with an increase in JNK signaling and cyclin D levels. Importantly, this deficit can be rescued by pharmacological JNK inhibition, suggesting that increased JNK activity due to loss of p38 $\alpha$ is specifically responsible for the inability of myoblasts to exit the cell cycle in order to begin differentiating (32). Mechanistically, p38 $\alpha$ has been shown to inhibit the JNK pathway by upregulating the MAPK phosphatase MKP-1 (also known as DUSP1; ref. 32). Altogether, p38 $\alpha / \beta$ positively regulates muscle cell differentiation on multiple levels and through many separate mechanisms.

Consequently, constitutive activation of p38 in myoblasts was shown to be sufficient to induce expression of differentiation markers and promote formation of multinucleated myotubes (23), whereas inhibition of p38 $\alpha / \beta$ prevents induction of muscle-specific genes and myoblast fusion (21-23). In two studies, treatment of myoblasts with the $\mathrm{p} 38 \alpha / \beta$ inhibitor SB203580 prevented formation of myotubes and expression of markers of both early and late myogenesis such as myogenin and creatine kinase, suggesting that $\mathrm{p} 38 \alpha / \beta$ plays an important role early in myogenesis $(21,22)$. Another study found that the $\mathrm{p} 38 \alpha / \beta$ inhibitor SB202190 blocked 
formation of myosin heavy chain-positive (MHC-positive) myotubes in multiple cell lines, including primary human myoblasts (23). In support of these studies, myoblasts derived from mice harboring genetic deletions in $\mathrm{p} 38 \alpha$ also fail to undergo differentiation.

p38 in satellite cell regulation. Satellite cells are resident muscle stem cells that provide muscle with its ability to regenerate and repair (33). These cells reside beneath the basal lamina of the myofiber and remain in a quiescent state - controlled by expression of the transcription factor Pax 7 - until activated by environmental cues, such as growth factors and cytokines, to proliferate as myoblasts $(34,35)$. Satellite cell activation leads to MyoD expression and subsequent reentry into the cell cycle (36-38). Like other stem cells, satellite cells can undergo asymmetric cell division such that one daughter is activated while the other remains quiescent, thereby renewing the stem cell pool (39). A small fraction of activated satellite cells retains stemness by downregulating MyoD, while the other fraction becomes committed progenitors by downregulating Pax7 (40). p38 provides a link between regenerative cues, MyoD induction, and Pax7 expression, thus controlling satellite cell activation and differentiation (Figure 2). Initially, p38 $\alpha / \beta$ was shown to be expressed in activated satellite cells, and $\mathrm{p} 38 \alpha / \beta$ inhibition caused satellite cells to exit the cell cycle and not undergo differentiation or respond to myogenic stimuli (41). This response was attributed to an inability of $\mathrm{p} 38 \alpha / \beta$ activity-deficient cells to properly induce MyoD expression (41). Also, it was later discovered that p38 is critical for Pax7 repression through recruitment of polycomb repressive complex 2 (PRC2) to the Pax 7 promoter (42). TNF- $\alpha$ activates p38 $\alpha$, which then phosphorylates the PRC2 subunit EZH2. Phosphorylated PRC2 interacts with the transcription factor YY1, which binds upstream of the Pax7 promoter to repress Pax7 expression, thus promoting cell cycle exit to quiescence (42). Interestingly, $\mathrm{p} 38 \alpha / \beta$ also appears to be essential for asymmetric stem cell fate determination of activated satellite cells. $\mathrm{p} 38 \alpha / \beta$ is activated in only one daughter cell, where it induces MyoD, thereby promoting division and differentiation. The other daughter cell, which lacks $\mathrm{p} 38 \alpha / \beta$, does not express MyoD and returns to a quiescent state (43). Thus, through the induction of MyoD and repression of Pax7, p38 promotes activation of quiescent satellite cells in response to myogenic stimuli.

p38 $\gamma$ regulates the decision for satellite cells to differentiate through phosphorylation of MyoD on Ser199 and Ser200, which promotes MyoD binding to the histone methyltransferase KMT1A. The MyoD-KMT1A repressive complex is recruited to the myogenin promoter, preventing expression and thereby blocking induction of the differentiation gene program (44). Consistent with this function, myoblasts from p38 $\gamma$-deficient mice have decreased ability to fuse into multinucleated, MHC-positive myotubes (32).

\section{p38 promotes inflammation}

p38 $\alpha$ promotes inflammation by facilitating the production of proinflammatory cytokines, such as IL-1 and TNF- $\alpha$ (45), and can activate transcription factors, such as AP-1 family members, that induce cytokine expression $(46,47)$. In addition, $\mathrm{p} 38 \alpha$ and $\mathrm{p} 38 \beta$ and their target MK2 promote cytokine expression by phosphorylating proteins that bind to AU-rich elements found in cytokine-encoding mRNA, thus stabilizing those transcripts $(31,48)$. Initial attempts to utilize $\mathrm{p} 38 \alpha / \beta$ inhibitors therapeutically in the clinic focused on inflammatory conditions, such as rheumatoid arthritis (RA) $(49,50)$, neuropathic pain (51), focal segmental glomerulosclerosis (52), and COPD (14). In some cases, although $\mathrm{p} 38 \alpha / \beta$ inhibitors were shown to be effective in preclinical models, they failed in clinical trials due to onset of unanticipated liver and neurological side effects $(50,53)$. Other inhibitors were shown to be safe at lower doses but conferred modest to no efficacy in humans $(54,55)$.

Inflammation also plays a major role in muscular dystrophy. Increased expression of cytokines and infiltration of immune cells are thought to be directly related to pathology $(56,57)$. For example, IL17 mRNA is increased in muscle biopsies from patients with Duchenne muscular dystrophy (DMD) (58). In $m d x$ mice, a mouse model of DMD, elevated IL-6 levels persist following injury compared with those in control mice and have been linked to muscle wasting (59). Moreover, steroid treatment to reduce inflammation is commonly used for DMD patients and effectively increases muscle strength while simultaneously delaying disease progression (60). p38 $\alpha / \beta$ inhibition has the potential to decrease inflammation in muscular dystrophy, although its lack of success as a treatment for inflammatory diseases indicates that other aspects of muscular dystrophy pathology will need to be targeted in order for $\mathrm{p} 38 \alpha / \beta$ inhibition to be an effective therapeutic. Below, we examine the potential of $\mathrm{p} 38 \alpha / \beta$ inhibition in treating several diseases that affect the muscle, with a particular focus on FSHD.

\section{p38 in skeletal muscle disease}

Most studies on p38 in muscle diseases to date have been conducted in preclinical models. For example, initial evidence for a role of p38 in DMD came from the finding that p38 is upregulated in exercise-trained 
$m d x$ mice, but not in exercise-trained WT mice or in nonexercised $m d x$ mice (61). The authors speculated that p38 has a role in degeneration of dystrophic muscle, as its upregulation was specific to exercise training, in which degeneration was exacerbated. Deletion of the dual-specificity phosphatase DUSP1, which normally acts to inhibit p38 activity, enhanced the dystrophic phenotype of $m d x$ mice by preventing regeneration (62). Pharmacological inhibition of $\mathrm{p} 38 \alpha / \beta$ improved the survival of $m d x$ mouse myofibers challenged with oxidative stress, providing evidence that $\mathrm{p} 38 \alpha / \beta$ inhibition could be used as a potential therapy for DMD (63). A comprehensive study by Wissing et al. found that $\mathrm{p} 38 \alpha$ deficiency in the $m d x$ mouse model and the sarcoglycan-knockout mouse $\left(\mathrm{Sgct}^{--}\right)$, a model for limb-girdle muscular dystrophy, alleviated pathology in both models, decreasing fibrosis and macrophage infiltration and improving endurance on a treadmill (64). p38 activation also appeared to be at least partially sufficient for development of some muscular dystrophy phenotypes, as artificially increasing p38 activity by expression of constitutively active MKK6 induced muscle degeneration pathology reminiscent of that observed in muscular dystrophies. It is worth noting that MKK6 expression likely increased p38 activity beyond physiological levels and could thus cause toxicity. The authors went on to demonstrate that these effects were due to p38 phosphorylating and activating the proapoptotic factor Bax (64). Taken together, these data suggest that p38 inhibition may be beneficial for treatment of multiple forms of muscular dystrophy by preventing inflammation and myofiber death.

\section{p38 inhibitors as a therapy for LMNA-related heart disease}

Inhibition of p38 $\alpha$ is currently being pursued as a potential treatment for LMNA-related dilated cardiomyopathy (DCM) (ClinicalTrials.gov, NCT02057341). This form of heart disease is caused by mutations in LMNA, which encodes nuclear A/C type lamins (65). Mutations in LMNA lead to biomechanical defects in cardiomyocytes due to loss of nuclear integrity, ultimately causing cardiac dilation and progressive heart failure (66). In contrast to the previously described studies where beneficial effects of $\mathrm{p} 38 \alpha / \beta$ inhibition were due to prevention of p38-mediated inflammation and apoptosis, p38 signaling has been shown to be proximal to the mutation that causes LMNA-related DCM. Analysis of the transcriptomes of heart tissue from a LMNA mutation-harboring mouse model pointed to hyperactivation of the p38 pathway prior to the onset of cardiac disease phenotypes (67). Treatment with a p38 $\alpha$ inhibitor prevented left-ventricle dilation and ameliorated cardiac phenotypes caused by LMNA mutations. The mechanism for this would later be proposed to be the mechanical correction of cytoskeletal defects upon p38 $\alpha$ inhibition (68). Three separate LMNA mutations were shown to cause mechanical defects in cardiomyocytes, including reduced cytoskeletal elasticity and adhesion, by use of atomic force microscopy (AFM). These biomechanical defects were rescued by treatment with a p38 $\alpha$ inhibitor (68). The authors proposed that $\mathrm{p} 38$ may be contributing to the altered biomechanical features of LMNA mutant cardiomyocytes via its established role in regulating the actin cytoskeleton, and thus these p38-mediated effects may have led to the observed loss of contractility and increased apoptosis (69-71). Indeed, cardiomyocytes harboring mutations in LMNA have a disorganized network of actin filaments, and treatment with a p38 $\alpha$ inhibitor restored them to a WT-like state (68). Thus, rather than preventing inflammation and apoptosis, p38 $\alpha$ inhibition may correct some of the mechanical flaws caused by defective lamin in cardiac cells due to genetic mutations. It is worth noting that patients with LMNA mutations can also present with varying degrees of skeletal myopathy, including Emery-Dreifuss muscular dystrophy and limb-girdle muscular dystrophy (72-74). It would be interesting to explore the potential of p38 $\alpha$ inhibitors to improve skeletal muscle phenotypes associated with LMNA mutations as well.

\section{p38 inhibitors as a multitargeted therapy for FSHD}

Inhibition of $\mathrm{p} 38 \alpha / \beta$ is also being pursued as a potential treatment for FSHD (for review, see ref. 75). FSHD is a progressive muscle degenerative disease that affects approximately 1:15,000 individuals (76). This dystrophy is typically diagnosed in the second or third decade of life, though a more-severe infantile form exists. Initially, muscles of the face, shoulder, and upper arms are affected; and as the disease progresses, it causes degeneration of muscles in the lower extremities, as well as loss of mobility and inability to perform daily tasks (75). There are 2 genetic forms of FSHD. FSHD1 is more common and is caused by a contraction in the number of D4Z4 macrosatellite repeats in the subtelomeric region of the $q$ arm of chromosome 4 (77). FSHD2 is caused by mutations in epigenetic regulators, most commonly the chromatin modifier SMCHD1 (78). Both mutations lead to inappropriate expression of DUX4, a transcription factor encoded within the D4Z4 repeats that is typically only expressed during early embryonic development, due to epigenetic de-repression of the D4Z4 region $(79,80)$. In cultured myoblasts isolated from FSHD patient biopsy specimens, only a small fraction of myonuclei express 
DUX4 following induced differentiation into myotubes (80). DUX4 expression in myonuclei leads to aberrant expression of many transcripts that are typically restricted to the cleavage-stage embryo (81-84). Additionally, DUX4 interacts with critical RNA-binding proteins involved in RNA splicing and translation, potentially altering their function (85). It is not yet understood whether DUX4 toxicity is due to misexpression of its transcriptional targets or dominant interactions with essential cellular proteins. However, the net result of DUX4 misexpression in FSHD myotubes or cells engineered to conditionally express DUX4 is induction of many cellular stresses - including oxidative stress (86-88), proteotoxic stress (89), and accumulation of double-stranded RNA (90) - that ultimately lead to the death of the myofiber. Precisely how DUX4 kills cells is also an area of active investigation. Multiple studies have found that DUX4 expression activates caspase-3/7 via a p53-dependent mechanism (91-93), but p53-independent cell death due to DUX4 expression has also been described (90, 94).

Pathological features of biopsy samples from affected muscle tissue of FSHD patients include degenerating and regenerating fibers; replacement of muscle with fatty-fibrotic tissue; and pronounced inflammation, particularly immune cell infiltration (95). Analysis of the transcriptome of affected muscle tissue in patients with FSHD revealed downregulation of genes involved in muscle development and differentiation, and upregulation of extracellular matrix, inflammatory, and immune response genes $(81,96)$. Thus, DUX4 expression causes FSHD-associated symptoms by inducing muscle fiber degeneration and promoting inflammation. How the immune system responds to FSHD muscles and contributes to pathology is an area of active investigation.

Inhibition of $p 38 \alpha / \beta$ decreases expression of DUX4, the FSHD disease gene. Interestingly, a screen for molecules that prevent DUX4 expression in FSHD patient-derived muscle cells uncovered multiple p38 $\alpha / \beta$ inhibitors as potent repressors of the DUX4 target gene MBD3L2 (97). Further examination of the effects of these compounds on FSHD patient-derived muscle cells led to the discovery that p38 inhibition causes a reduction in DUX4 mRNA that in turn results in decreased mRNA levels of several DUX4 target genes $(97,98)$. Importantly, p38 $\alpha / \beta$ inhibition decreased cell death in FSHD patient cells (97). As DUX4 is a primate-specific gene, animal models of FSHD are lacking. However, p38 $\alpha / \beta$ inhibitor treatment of humanized mice with FSHD patient-derived muscle xenografts reduced DUX4 expression, target gene expression, and apoptosis in xenografted muscle (98). Notably, FSHD xenografts are generated in immune-deficient mice and thus fail to model the role of immune cell-mediated inflammation, which may also provide a treatment mode for $\mathrm{p} 38 \alpha / \beta$ inhibitors. Thus, $\mathrm{p} 38 \alpha / \beta$ inhibition in preclinical models of FSHD appears to be targeting the root cause of the disease by decreasing DUX4 expression. These data provided the basis for clinical development of p38 inhibitors for treating FSHD, which is discussed below.

Currently, the mechanism by which p38 controls DUX4 expression in muscle is not fully understood. DUX4 is not expressed in proliferating FSHD patient myoblasts in cell culture, and its expression is increased in only a small fraction of myotube nuclei following myotube differentiation (80, 83). p38 $\alpha / \beta$ inhibitors block DUX4 mRNA expression as well as disrupting MyoD/MRF transcription factor function, as described above, supporting a role for p38 in modulating DUX4 expression as part of the differentiation transcriptional regulatory response (Figure 2). It is worth noting that treatments with concentrations of $\mathrm{p} 38 \alpha / \beta$ inhibitors up to $1 \mu \mathrm{M}$ still inhibited DUX4 expression but had no effect on the formation of myotubes or expression of differentiation markers $(97,98)$. However, earlier studies showing $\mathrm{p} 38 \alpha / \beta$ inhibition of myogenesis used inhibitor concentrations that were between 5- and 20-fold higher, indicating that inhibition of differentiation and DUX4 expression is concentration dependent (21-23). Furthermore, RNA sequencing (RNA-Seq) analysis of FSHD myotubes treated with 2 different $p 38 \alpha / \beta$ inhibitors revealed that the majority of statistically significant changes in gene expression consisted of reduced levels of DUX4 target genes, not myogenic transcripts (97). In this experiment, only a single time point later in differentiation was analyzed, leaving the possibility that $\mathrm{p} 38 \alpha / \beta$ inhibition causes delays in myogenic differentiation. A recent CRISPR genome screen for DUX4 toxicity reported that DUX4 function is also controlled by hypoxia signaling (99). DUX4 expression itself leads to induction of hypoxia, as measured by stabilization of HIF-1, the hypoxic response master regulator. Interestingly, pharmacological inhibition of hypoxia signaling decreased DUX4 protein stability, DUX4 expression targets, and apoptosis. Several studies have demonstrated that p38 regulates hypoxia signaling (100-102), so perhaps this is one mechanism by which p38 $\alpha / \beta$ inhibition decreases DUX4-dependent gene expression. Alternatively, p38 could regulate transcription factors or chromatin modifiers that control the epigenetic status of the D4Z4 repeat locus in which DUX4 is expressed from the terminal repeat. For example, the polycomb repressive complex (PRC) epigenetically represses the D4Z4 repeat by maintaining repressive $\mathrm{H} 3 \mathrm{~K} 27 \mathrm{me} 3$ marks, and the PRC subunit $\mathrm{EZH} 2$ is a known target of $\mathrm{p} 38 \alpha$ in satellite cells $(103,104)$ (Figure 2). It will be interesting to determine 
whether other DUX4-repressing proteins are targets of $\mathrm{p} 38 \alpha / \beta$ inhibitors in muscle to further understand whether p38 controls DUX4 expression primarily through epigenetic repressors.

Additional potential therapeutic effects of $p 38 \alpha / \beta$ inhibition in FSHD. p38 plays a critical role in controlling myogenesis (highlighted above); therefore, any potential side effects of pharmacological $\mathrm{p} 38 \alpha / \beta$ inhibition that affect satellite cell regulation or myogenic differentiation are of concern. In particular, chronic inhibition of p38 $\alpha / \beta$ has the potential to result in a reduction in the ability of satellite cells to proliferate as myoblasts and further differentiate to repair and replace damaged myofibers. The effects of long-term treatment with p38 inhibitors on muscle regeneration have yet to be fully explored. As muscle in patients with FSHD shows evidence of active regeneration based on expression of embryonic isoforms of myosin and the presence of centrally located nuclei, chronic $\mathrm{p} 38 \alpha / \beta$ inhibition as a long-term treatment may not be feasible (105). As noted above, at low concentrations, these inhibitors did not affect early stages of myogenesis in FSHD xenograft muscle studies $(97,98)$. Monitoring the effects of inhibitors on muscle physiology and regeneration during clinical testing of $\mathrm{p} 38 \alpha / \beta$ inhibitors in FSHD will be important for evaluating the expected long-term treatment plan needed for therapeutically effective use of these inhibitors, as previous trials with p38 $\alpha / \beta$ inhibitors for other nonmuscle indications did not specifically examine muscle toxicology.

Similar to other muscular dystrophies where $\mathrm{p} 38 \alpha / \beta$ inhibition has been tested in preclinical models (64), FSHD is associated with significant inflammation and a distinct innate and adaptive immune signature (106). Therefore, $\mathrm{p} 38 \alpha / \beta$ inhibitors may provide additional benefits for patients with FSHD independent of DUX4 repression by targeting and preventing inflammation and Bax-dependent apoptosis, as has been observed in $m d x$ and $\mathrm{Sgct}^{-1-}$ mouse models of muscular dystrophy (64). Preclinical models of FSHD inflammation that would enable this hypothesis to be tested are currently under development and will be valuable tools for future FSHD clinical trial development. It is also now possible to gain information on the infiltrating immune cell types in FSHD muscle during disease progression using high-spatial-resolution RNA-Seq and antibody protein mapping of muscle (107). These methods could be of value for analyzing the effects of therapies on inflammation in FSHD clinical trials. Additionally, p38 $\alpha / \beta$ inhibitors are known to prevent p53-dependent apoptosis. Mechanistic studies have shown that p38 directly phosphorylates p53 in response to viral infection and DNA damage caused by either UV radiation or chemotherapy, thereby promoting p53-dependent transcription and apoptosis $(108-110)$, another indication that $\mathrm{p} 38 \alpha / \beta$ is a favorable target for inhibition in FSHD therapy (91-93).

Losmapimod clinical trial and a future outlook. Among several $\mathrm{p} 38 \alpha / \beta$ inhibitors demonstrated to be efficacious in blocking DUX4 functions in cells from patients with FSHD, the $\mathrm{p} 38 \alpha / \beta$ inhibitor losmapimod was selected for clinical trials in part due to its positive clinical safety profile in humans. In a trial for COPD, losmapimod was well tolerated in more than 3500 participants $(54,55)$ but failed to demonstrate efficacy. More recently, a phase I study evaluated losmapimod in FSHD patients. There was a dose-dependent increase in losmapimod exposure in muscle, as well as evidence of target engagement, as measured by levels of phosphorylated Hsp27, an indirect target and surrogate marker of p38 function. Losmapimod accumulated to a concentration of approximately 100 $\mathrm{nM}$ in muscle, with a dosing regimen of $15 \mathrm{mg}$ per day (111), a level that was sufficient to inhibit DUX4 function in patient cell assays (97). The ReDUX4 phase II trial (ClinicalTrials.gov, NCT04003974) is currently ongoing, with results expected to be presented at the FSHD Society International Research Congress, June 24-25, 2021. The primary end point for this study is robust downregulation of DUX4-activated gene expression as measured by RNA levels of several DUX4 target genes in MRI-guided muscle biopsies collected after 16 and 36 weeks of treatment. Clinical measurements of mobility and muscle strength will also be made. Interim data showed little difference in DUX4-activated gene expression between placebo-treated and losmapimod-treated groups when looking at all trial participants; however, a decrease in DUX4-activated gene expression was observed in losmapimod-treated participants with the highest baseline levels of DUX4-activated gene expression (112).

The lack of relevant animal models to test the action of losmapimod on the multiple p38 targets, apart from the human xenograft model for DUX4, limits development of clinical trial protocols, but such information can be generated as part of future $\mathrm{p} 38 \alpha / \beta$ inhibitor trials. It remains to be determined what levels of DUX4 inhibition are required for effective clinical outcomes, and whether low levels of DUX4 expression are sufficient or if expression must be entirely eliminated to achieve a reasonable outcome for improvement of patient quality of life. It also remains to be determined whether $\mathrm{p} 38 \alpha / \beta$ inhibitor treatment will significantly reverse muscle damage or merely prevent further disease progression. The results of the ongoing losmapimod trial will provide an indication of the potential of $\mathrm{p} 38 \alpha / \beta$ inhibitors as therapeutics for FSHD. Moreover, the results will provide an invaluable molecular and clinical platform to enable future FSHD clinical trials, offering hope for treatment of this devastating disease. 


\section{Acknowledgments}

We would like to thank Seng Cheng and John Murphy for critical reading of the manuscript and Molly Bird for thoughtful discussions. We would also like to thank the Pfizer Worldwide Research, Development and Medical (WRDM), Postdoctoral Program for their support. CPE received grant support from the National Institute of Child Health and Human Development (NICHD) Paul D. Wellstone Muscular Dystrophy Cooperative Research Center (P50 HD060848).

Address correspondence to: Nicolas.Christoforou, Pfizer Inc., 610 Main St., Cambridge, Massachusetts 02139, USA. Phone: 857.324.9888; Email: Christoforou@Pfizer.com.

1. Morrison DK. MAP kinase pathways. Cold Spring Harb Perspect Biol. 2012;4(11):a011254.

2. Nebreda AR, Porras A. p38 MAP kinases: beyond the stress response. Trends Biochem Sci. 2000;25(6):257-260.

3. Cuadrado A, Nebreda AR. Mechanisms and functions of p38 MAPK signalling. Biochem J. 2010;429(3):403-417.

4. Carithers LJ, et al. A novel approach to high-quality postmortem tissue procurement: the GTEx Project. Biopreserv Biobank. 2015;13(5):311-319.

5. Wang Z, et al. The structure of mitogen-activated protein kinase p38 at 2.1-A resolution. Proc Natl Acad Sci U S A. 1997;94(6):2327-2332.

6. Chang CI, et al. Crystal structures of MAP kinase p38 complexed to the docking sites on its nuclear substrate MEF2A and activator MKK3b. Mol Cell. 2002;9(6):1241-1249.

7. Dolado I, et al. p38alpha MAP kinase as a sensor of reactive oxygen species in tumorigenesis. Cancer Cell. 2007;11(2):191-205.

8. Zhuang ZH, et al. Regulation of Drosophila p38 activation by specific MAP2 kinase and MAP3 kinase in response to different stimuli. Cell Signal. 2006;18(4):441-448.

9. Tong L, et al. A highly specific inhibitor of human p38 MAP kinase binds in the ATP pocket. Nat Struct Biol. 1997;4(4):311-316.

10. Jiang Y, et al. Characterization of the structure and function of a new mitogen-activated protein kinase (p38beta). J Biol Chem. 1996;271(30):17920-17926.

11. Lee JC, et al. Inhibition of monocyte IL-1 production by the anti-inflammatory compound, SK\&amp; F 86002. Int J Immunopharmacol. 1988;10(7):835-843.

12. Lee JC, et al. A protein kinase involved in the regulation of inflammatory cytokine biosynthesis. Nature. 1994;372(6508):739-746

13. Lee JC, et al. Inhibition of p38 MAP kinase as a therapeutic strategy. Immunopharmacology. 2000;47(2-3):185-201.

14. Norman P. Investigational p38 inhibitors for the treatment of chronic obstructive pulmonary disease. Expert Opin Investig Drugs. 2015;24(3):383-392.

15. Segalés J, et al. Regulation of muscle stem cell functions: a focus on the p38 MAPK signaling pathway. Front Cell Dev Biol. $2016 ; 4: 91$.

16. Lluís F, et al. Regulation of skeletal muscle gene expression by p38 MAP kinases. Trends Cell Biol. 2006;16(1):36-44.

17. Berkes CA, Tapscott SJ. MyoD and the transcriptional control of myogenesis. Semin Cell Dev Biol. 2005;16(4-5):585-595.

18. Puri PL, Sartorelli V. Regulation of muscle regulatory factors by DNA-binding, interacting proteins, and post-transcriptional modifications. J Cell Physiol. 2000;185(2):155-173.

19. Wang LH, Baker NE. E proteins and ID proteins: helix-loop-helix partners in development and disease. Dev Cell. 2015;35(3):269-280.

20. Pon JR, Marra MA. MEF2 transcription factors: developmental regulators and emerging cancer genes. Oncotarget. 2016;7(3):2297-2312.

21. Cuenda A, Cohen P. Stress-activated protein kinase-2/p38 and a rapamycin-sensitive pathway are required for C2C12 myogenesis. J Biol Chem. 1999;274(7):4341-4346.

22. Zetser A, et al. p38 mitogen-activated protein kinase pathway promotes skeletal muscle differentiation. Participation of the Mef2c transcription factor. J Biol Chem. 1999;274(8):5193-5200.

23. Wu Z, et al. p38 and extracellular signal-regulated kinases regulate the myogenic program at multiple steps. Mol Cell Biol. 2000;20(11):3951-3964.

24. Han J, et al. Activation of the transcription factor MEF2C by the MAP kinase p38 in inflammation. Nature. 1997;386(6622):296-299.

25. Zhao M et al. Regulation of the MEF2 family of transcription factors by p38. Mol Cell Biol. 1999;19(1):21-30.

26. Lluís F, et al. E47 phosphorylation by p38 MAPK promotes MyoD/E47 association and muscle-specific gene transcription. EMBO J. 2005;24(5):974-984.

27. Liu QC, et al. Comparative expression profiling identifies differential roles for Myogenin and p38 $\alpha$ MAP signaling in myogenesis J Mol Cell Biol. 2012;4(6):386-397.

28. Simone C, et al. p38 pathway targets SWI-SNF chromatin-remodeling complex to muscle-specific loci. Nat Genet. 2004;36(7):738-743.

29. Debril MB, et al. Transcription factors and nuclear receptors interact with the SWI/SNF complex through the BAF60c subunit. J Biol Chem. 2004;279(16):16677-16686.

30. Segalés J, et al. Chromatin-wide and transcriptome profiling integration uncovers p38 $\alpha$ MAPK as a global regulator of skeletal muscle differentiation. Skelet Muscle. 2016;6(1):9-15.

31. Briata P, et al. p38-dependent phosphorylation of the mRNA decay-promoting factor KSRP controls the stability of select myogenic transcripts. Mol Cell. 2005;20(6):891-903.

32. Perdiguero E, et al. Genetic analysis of p38 MAP kinases in myogenesis: fundamental role of p38alpha in abrogating myoblast proliferation. EMBO J. 2007;26(5):1245-1256.

33. Yin H, et al. Satellite cells and the muscle stem cell niche. Physiol Rev. 2013;93(1):23-67.

34. Buckingham M, Relaix F. PAX3 and PAX7 as upstream regulators of myogenesis. Semin Cell Dev Biol. 2015;44:115-125.

35. Seale P, Rudnicki MA. A new look at the origin, function, and "stem-cell" status of muscle satellite cells. Dev Biol. 2000;218(2):115-124

36. Füchtbauer EM, Westphal H. MyoD and myogenin are coexpressed in regenerating skeletal muscle of the mouse. Dev Dyn. 1992;193(1):34-39.

37. Yablonka-Reuveni Z, Rivera AJ. Temporal expression of regulatory and structural muscle proteins during myogenesis of satellite cells on isolated adult rat fibers. Dev Biol. 1994;164(2):588-603. 
38. Cooper RN, et al. In vivo satellite cell activation via Myf5 and MyoD in regenerating mouse skeletal muscle. J Cell Sci. 1999;112 (Pt 17):2895-2901.

39. Kuang S, et al. Asymmetric self-renewal and commitment of satellite stem cells in muscle. Cell. 2007;129(5):999-1010.

40. Zammit PS, et al. Muscle satellite cells adopt divergent fates: a mechanism for self-renewal? J Cell Biol. 2004;166(3):347-357.

41. Jones NC, et al. The p38alpha/beta MAPK functions as a molecular switch to activate the quiescent satellite cell. $J$ Cell Biol. 2005;169(1):105-116.

42. Palacios D, et al. TNF/p38 / polycomb signaling to Pax7 locus in satellite cells links inflammation to the epigenetic control of muscle regeneration. Cell Stem Cell. 2010;7(4):455-469.

43. Troy A, et al. Coordination of satellite cell activation and self-renewal by Par-complex-dependent asymmetric activation of p38a/ $\beta$ MAPK. Cell Stem Cell. 2012;11(4):541-553.

44. Gillespie MA, et al. p38-\{gamma\}-dependent gene silencing restricts entry into the myogenic differentiation program. J Cell Biol. 2009;187(7):991-1005

45. Cuenda A, Rousseau S. p38 MAP-kinases pathway regulation, function and role in human diseases. Biochim Biophys Acta. 2007;1773(8):1358-1375.

46. Garcia J, et al. A Mycoplasma fermentans-derived synthetic lipopeptide induces AP-1 and NF-kappaB activity and cytokine secretion in macrophages via the activation of mitogen-activated protein kinase pathways. J Biol Chem. 1998;273(51):34391-34398.

47. Yang Y, et al. Functional roles of p38 mitogen-activated protein kinase in macrophage-mediated inflammatory responses. Mediators Inflamm. 2014;2014:352371.

48. Neininger A, et al. MK2 targets AU-rich elements and regulates biosynthesis of tumor necrosis factor and interleukin-6 independently at different post-transcriptional levels. J Biol Chem. 2002;277(5):3065-3068.

49. Cohen SB, et al. Evaluation of the efficacy and safety of pamapimod, a p38 MAP kinase inhibitor, in a double-blind, methotrexatecontrolled study of patients with active rheumatoid arthritis. Arthritis Rheum. 2009;60(2):335-344.

50. Genovese MC, et al. A 24-week, randomized, double-blind, placebo-controlled, parallel group study of the efficacy of oral SCIO469, a p38 mitogen-activated protein kinase inhibitor, in patients with active rheumatoid arthritis. J Rheumatol. 2011;38(5):846-854.

51. Ostenfeld T, et al. A randomized, placebo-controlled trial of the analgesic efficacy and safety of the p38 MAP kinase inhibitor, losmapimod, in patients with neuropathic pain from lumbosacral radiculopathy. Clin J Pain. 2015;31(4):283-293.

52. Gipson DS, et al. Assessing the impact of losmapimod on proteinuria in idiopathic focal segmental glomerulosclerosis. Kidney Int Rep. 2020;5(8):1228-1239.

53. Genovese MC. Inhibition of p38: has the fat lady sung? Arthritis Rheum. 2009;60(2):317-320.

54. Watz H, et al. Efficacy and safety of the p38 MAPK inhibitor losmapimod for patients with chronic obstructive pulmonary disease: a randomised, double-blind, placebo-controlled trial. Lancet Respir Med. 2014;2(1):63-72.

55. Fisk M, et al. The p38 mitogen activated protein kinase inhibitor losmapimod in chronic obstructive pulmonary disease patients with systemic inflammation, stratified by fibrinogen: a randomised double-blind placebo-controlled trial. PLoS One. 2018;13(3):e0194197.

56. Rosenberg AS, et al. Immune-mediated pathology in Duchenne muscular dystrophy. Sci Transl Med. 2015;7(299):299rv4.

57. Londhe P, Guttridge DC. Inflammation induced loss of skeletal muscle. Bone. 2015;80:131-142.

58. De Pasquale L, et al. Increased muscle expression of interleukin-17 in Duchenne muscular dystrophy. Neurology. 2012;78(17):1309-1314.

59. Kurek JB, et al. Leukemia inhibitory factor and interleukin-6 are produced by diseased and regenerating skeletal muscle. Muscle Nerve. 1996;19(10):1291-1301.

60. Moxley RT, et al. Practice parameter: corticosteroid treatment of Duchenne dystrophy: report of the Quality Standards Subcommittee of the American Academy of Neurology and the Practice Committee of the Child Neurology Society. Neurology. 2005;64(1):13-20.

61. Nakamura A, et al. Up-regulation of mitogen activated protein kinases in mdx skeletal muscle following chronic treadmill exercise. Biochim Biophys Acta. 2005;1740(3):326-331.

62. Shi H, et al. MAP kinase phosphatase-1 deficiency impairs skeletal muscle regeneration and exacerbates muscular dystrophy. FASEB J. 2010;24(8):2985-2997

63. Smythe GM, Forwood JK. Altered mitogen-activated protein kinase signaling in dystrophic (mdx) muscle. Muscle Nerve. 2012;46(3):374-383.

64. Wissing ER, et al. P38 $\alpha$ MAPK underlies muscular dystrophy and myofiber death through a Bax-dependent mechanism. Hum Mol Genet. 2014;23(20):5452-5463.

65. Fatkin D, et al. Missense mutations in the rod domain of the lamin A/C gene as causes of dilated cardiomyopathy and conductionsystem disease. $N$ Engl J Med. 1999;341(23):1715-1724.

66. Goidescu CM. Dilated cardiomyopathy produced by lamin A/C gene mutations. Clujul Med. 2013;86(4):309-312.

67. Muchir A, et al. Abnormal p38 $\alpha$ mitogen-activated protein kinase signaling in dilated cardiomyopathy caused by lamin A/C gene mutation. Hum Mol Genet. 2012;21(19):4325-4333.

68. Laurini E, et al. Biomechanical defects and rescue of cardiomyocytes expressing pathologic nuclear lamins. Cardiovasc Res. 2018;114(6):846-857.

69. Guay J, et al. Regulation of actin filament dynamics by p38 map kinase-mediated phosphorylation of heat shock protein 27. J Cell Sci. 1997;110(Pt 3):357-368.

70. Zhang W, Liu HT. MAPK signal pathways in the regulation of cell proliferation in mammalian cells. Cell Res. 2002;12(1):9-18

71. Communal C, et al. Functional consequences of caspase activation in cardiac myocytes. Proc Natl Acad Sci U S A. 2002;99(9):6252-6256.

72. Bonne G, et al. Mutations in the gene encoding lamin A/C cause autosomal dominant Emery-Dreifuss muscular dystrophy. Nat Genet. 1999;21(3):285-288.

73. Brodsky GL, et al. Lamin A/C gene mutation associated with dilated cardiomyopathy with variable skeletal muscle involvement Circulation. 2000;101(5):473-476.

74. Muchir A, et al. Identification of mutations in the gene encoding lamins A/C in autosomal dominant limb girdle muscular dystrophy with atrioventricular conduction disturbances (LGMD1B). Hum Mol Genet. 2000;9(9):1453-1459.

75. DeSimone AM, et al. Facioscapulohumeral muscular dystrophy. Compr Physiol. 2017;7(4):1229-1279.

76. Wagner KR. Facioscapulohumeral muscular dystrophies. Continuum (Minneap Minn). 2019;25(6):1662-1681.

77. Lunt PW, et al. Correlation between fragment size at D4F104S1 and age at onset or at wheelchair use, with a possible generational 
effect, accounts for much phenotypic variation in 4q35-facioscapulohumeral muscular dystrophy (FSHD). Human Molecular Genetics. 1995;4(5):951-958.

78. de Greef JC, et al. Common epigenetic changes of D4Z4 in contraction-dependent and contraction-independent FSHD. Hum Mutat. 2009;30(10):1449-1459.

79. Lemmers RJLF, et al. Facioscapulohumeral muscular dystrophy is uniquely associated with one of the two variants of the $4 \mathrm{q}$ subtelomere. Nat Genet. 2002;32(2):235-236.

80. Snider L, et al. Facioscapulohumeral dystrophy: incomplete suppression of a retrotransposed gene. PLoS Genet. 2010;6(10):e1001181.

81. Rahimov F, et al. Transcriptional profiling in facioscapulohumeral muscular dystrophy to identify candidate biomarkers. Proc Natl Acad Sci U S A. 2012;109(40):16234-16239.

82. Geng LN, et al. DUX4 activates germline genes, retroelements, and immune mediators: implications for facioscapulohumeral dystrophy. Dev Cell. 2012;22(1):38-51.

83. Rickard AM, et al. Endogenous DUX4 expression in FSHD myotubes is sufficient to cause cell death and disrupts RNA splicing and cell migration pathways. Hum Mol Genet. 2015;24(20):5901-5914.

84. Jagannathan S, et al. Model systems of DUX4 expression recapitulate the transcriptional profile of FSHD cells. Hum Mol Genet. 2016;25(20):4419-4431.

85. Ansseau E, et al. Homologous transcription factors DUX4 and DUX4c associate with cytoplasmic proteins during muscle differentiation PLoS One. 2016;11(1):e0146893.

86. Winokur ST, et al. Expression profiling of FSHD muscle supports a defect in specific stages of myogenic differentiation. Hum Mol Genet. 2003;12(22):2895-2907.

87. Dmitriev P, et al. DUX4-induced constitutive DNA damage and oxidative stress contribute to aberrant differentiation of myoblasts from FSHD patients. Free Radic Biol Med. 2016;99:244-258

88. Turki A, et al. Functional muscle impairment in facioscapulohumeral muscular dystrophy is correlated with oxidative stress and mitochondrial dysfunction. Free Radic Biol Med. 2012;53(5):1068-1079.

89. Homma S, et al. Expression of FSHD-related DUX4-FL alters proteostasis and induces TDP-43 aggregation. Ann Clin Transl Neurol. 2015;2(2):151-166.

90. Shadle SC, et al. DUX4-induced dsRNA and MYC mRNA stabilization activate apoptotic pathways in human cell models of facioscapulohumeral dystrophy. PLoS Genet. 2017;13(3):e1006658.

91. Bosnakovski D, et al. An isogenetic myoblast expression screen identifies DUX4-mediated FSHD-associated molecular pathologies. EMBO J. 2008;27(20):2766-2779.

92. Wallace LM, et al. DUX4, a candidate gene for facioscapulohumeral muscular dystrophy, causes p53-dependent myopathy in vivo. Ann Neurol. 2010;69(3):540-552.

93. Kowaljow V, et al. The DUX4 gene at the FSHD1A locus encodes a pro-apoptotic protein. Neuromuscul Disord. 2007;17(8):611-623.

94. Bosnakovski D, et al. The DUX4 homeodomains mediate inhibition of myogenesis and are functionally exchangeable with the Pax7 homeodomain. J Cell Sci. 2017;130(21):3685-3697.

95. Statland JM, Tawil R. Facioscapulohumeral muscular dystrophy. Continuum (Minneap Minn). 2016;22(6):1916-1931.

96. Wang LH, et al. MRI-informed muscle biopsies correlate MRI with pathology and DUX4 target gene expression in FSHD. Hum Mol Genet. 2019;28(3):476-486.

97. Rojas LA, et al. p38 $\alpha$ regulates expression of DUX4 in a model of facioscapulohumeral muscular dystrophy. J Pharmacol Exp Ther. 2020;374(3):489-498.

98. Oliva J, et al. Clinically advanced p38 inhibitors suppress DUX4 expression in cellular and animal models of facioscapulohumeral muscular dystrophy. J Pharmacol Exp Ther. 2019;370(2):219-230.

99. Lek A, et al. Applying genome-wide CRISPR-Cas9 screens for therapeutic discovery in facioscapulohumeral muscular dystrophy. Sci Transl Med. 2020;12(536):eaay0271.

100. Conrad PW, et al. Selective activation of p38alpha and p38gamma by hypoxia. Role in regulation of cyclin D1 by hypoxia in PC12 cells. J Biol Chem. 1999;274(33):23570-23576.

101. Xu L, et al. Hypoxia-induced activation of p38 mitogen-activated protein kinase and phosphatidylinositol 3'-kinase signaling pathways contributes to expression of interleukin 8 in human ovarian carcinoma cells. Clin Cancer Res. 2004;10(2):701-707.

102. Park EC, Rongo $\mathrm{C}$. The $\mathrm{p} 38 \mathrm{MAP}$ kinase pathway modulates the hypoxia response and glutamate receptor trafficking in aging neurons. Elife. 2016;5:1822.

103. Himeda CL, et al. Facioscapulohumeral muscular dystrophy as a model for epigenetic regulation and disease. Antioxid Redox Signal. 2015;22(16):1463-1482.

104. Mozzetta C, et al. Selective control of Pax7 expression by TNF-activated p38 / polycomb repressive complex 2 (PRC2) signaling during muscle satellite cell differentiation. Cell Cycle. 2011;10(2):191-198.

105. Banerji CRS, et al. Skeletal muscle regeneration in facioscapulohumeral muscular dystrophy is correlated with pathological severity. Hum Mol Genet. 2020;29(16):2746-2760.

106. Yao Z, et al. DUX4-induced gene expression is the major molecular signature in FSHD skeletal muscle. Hum Mol Genet. 2014;23(20):5342-5352.

107. Merritt CR, et al. Multiplex digital spatial profiling of proteins and RNA in fixed tissue. Nat Biotechnol. 2020;38(5):586-599.

108. Bulavin DV, et al. Phosphorylation of human p53 by p38 kinase coordinates N-terminal phosphorylation and apoptosis in response to UV radiation. EMBO J. 1999;18(23):6845-6854.

109. Sanchez-Prieto R, et al. A role for the p38 mitogen-acitvated protein kinase pathway in the transcriptional activation of p53 on genotoxic stress by chemotherapeutic agents. Cancer Res. 2000;60(9):2464-2472.

110. Perfettini JL, et al. Essential role of p53 phosphorylation by p38 MAPK in apoptosis induction by the HIV-1 envelope. J Exp Med. 2005;201(2):279-289.

111. Fulcrum Therapeutics. Fulcrum Therapeutics Announced Results of Phase 1 Clinical Trial of Losmapimod in FSHD. https:// ir.fulcrumtx.com/node/6656/pdf. Updated October 4, 2019. Accessed February 16, 2021.

112. Fulcrum Therapeutics. Fulcrum Therapeutics Announces Interim Analysis Data from its ReDUX4 Trial in Facioscapulohumeral Muscular Dystrophy (FSHD). https://ir.fulcrumtx.com/node/7386/pdf. Updated August 11, 2020. Accessed February 16, 2021. 